\title{
Article \\ Some Examples of Calculation of Massless and Massive Feynman Integrals
}

\author{
Anatoly V. Kotikov \\ Bogoliubov Laboratory of Theoretical Physics, Joint Institute for Nuclear Research, 141980 Dubna, Russia; \\ kotikov@theor.jinr.ru
}

check for

updates

Citation: Kotikov, A.V. Some Examples of Calculation of Massless and Massive Feynman Integrals. Particles 2021, 4, 361-380. https: / / doi.org/10.3390/particles4030031

Academic Editor: Armen Sedrakian

Received: 19 July 2021

Accepted: 10 August 2021

Published: 14 August 2021

Publisher's Note: MDPI stays neutral with regard to jurisdictional claims in published maps and institutional affiliations.

Copyright: (C) 2021 by the author. Licensee MDPI, Basel, Switzerland. This article is an open access article distributed under the terms and conditions of the Creative Commons Attribution (CC BY) license (https:/ / creativecommons.org/licenses/by/ $4.0 /)$.
Abstract: We show some examples of calculations of massless and massive Feynman integrals.

Keywords: Feynman integrals; dimensional regularization; singularities

\section{Introduction}

At present, the calculation of the Feynman integrals (FIs) provides basic information, both on the properties of the experimentally investigated processes and on the characteristics of the physical models under study. Calculations of the matrix elements of the cross sections of the processes under study depend on the internal properties of particles participating in the interactions, such as masses, spins, etc., and, strictly speaking, require the calculation of Feynman integrals, including those with massive propagators. Depending on the kinematics of the processes under study, the values of some masses can be neglected. Studying the characteristics of physical models (for example, critical parameters, anomalous particle sizes and operators) usually requires the calculation of massless Feynman integrals, which have a much simpler structure. This allows obtaining results for these characteristics in high orders of the perturbation theory.

I would like to draw your attention to the fact that, when calculating FIs, it is recommended to use analytical methods whenever possible. The point is that the numerical calculation of FIs is severely limited due to the singularities arising in them, and also (especially for gauge theories) due to strong mutual cancellations between contributions from different diagrams or even between parts of the same diagram.

Note that when using the dimensional regularization [1-4], i.e., when calculating the FIs for an arbitrary dimension of space, once found diagrams for some model of a field theory (or process) can be applied to other models (or processes), since the main object of study is the so-called scalar master integrals. Consequently, the complexity of analytical calculations of FI is compensated by their versatility as applied to various quantum field models.

Note also the fact that the calculation of complicated diagrams may be of some independent interest. For example, the use of non-trivial identities, such as the "uniqueness" relation [5,6], can provide information (see [7-13]) about the properties of some integrals and series which are not yet in the reference literature. For example, the calculations of the same Feynman integral carried out in [12-14], using various methods, have made it possible to find a previously unknown relationship between hypergeometric functions with arguments 1 and -1 . This relation has been neatly proven quite recently [15].

Recently, many powerful original methods for calculating Feynman integrals have appeared (see, for example, recent reviews in [16,17]), which are often inferior in breadth of application to standard methods, such as the $\alpha$-representation and the Feynman parameter technique (see, for example, $[18,19])$, however, can significantly increase the computation accuracy for a limited set of quantities (or processes). 
This short article is devoted to the consideration of two FIs, one with massless propagators, and the other with massive propagators, the calculations of which just demonstrate the effectiveness of modern methods for calculating Feynman diagrams.

In the massless case, we will consider a single 5-loop master diagram that contributes to the $\beta$-function of the $\varphi^{4}$-model. In the initial calculations [20] of the 5-loop correction to the $\beta$-function of the $\varphi^{4}$-model, the results of four FIs were found only numerically. Their analytical results were obtained by Kazakov (see $[7,8,12,13])$, but they have been published without any intermediate results. Moreover, all calculations were performed in $x$-space, which can make them difficult to understand. Recently, two of the four diagrams have been exactly recalculated in [16] in $p$-space and are presented with intermediate calculations. In Section 3, we provide a neat calculation for the third diagram.

In the massive case, in Section 5, we consider the computation of one of masterintegrals [21] contributing to the relationship between the $\overline{M S}$-mass and the pole-mass of the Higgs boson in the standard model in the limit of heavy Higgs. Results for the master-integral, along with the results for other master-integrals, were calculated [22] in the early 2000s, but unfortunately they have not been published. Some sets of variables for integration are presented in Appendix A.

\section{Basic Formulas for Massless Diagrams}

Let us briefly consider the rules for calculation of massless diagrams. All calculations are carried out in momentum space with $d=4-2 \varepsilon$.

Propagator is represented as

$$
\frac{1}{\left(q^{2}\right)^{\alpha}} \equiv \frac{1}{q^{2 \alpha}}=\underset{\vec{q}}{\rightarrow} \quad \alpha \quad \bullet
$$

where $\alpha$ is called the line index.

The following formulas hold:

A. For simple chain:

$$
\frac{1}{q^{2 \alpha_{1}}} \frac{1}{q^{2 \alpha_{2}}}=\frac{1}{q^{2\left(\alpha_{1}+\alpha_{2}\right)}},
$$

or graphically

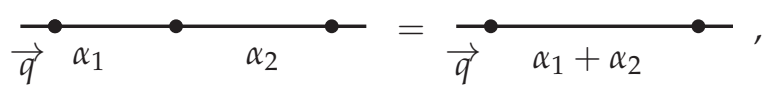

i.e., the product of propagators is equivalent to a new propagator with an index equal to the sum of the indices of the original propagators.

B. A simple loop can be integrated as

$$
\int \frac{D k \mu^{2 \varepsilon}}{(q-k)^{2 \alpha_{1}} k^{2 \alpha_{2}}}=N_{d} \frac{\mu^{2 \varepsilon}}{q^{2\left(\alpha_{1}+\alpha_{2}-d / 2\right)}} A\left(\alpha_{1}, \alpha_{2}\right),
$$

where

$$
D k=\frac{d^{d} k}{(2 \pi)^{d}}
$$

is usual integration in Euclidean measure and

$$
N_{d}=\frac{1}{(4 \pi)^{d / 2}}, \quad A(\alpha, \beta)=\frac{a(\alpha) a(\beta)}{a(\alpha+\beta-d / 2)}, a(\alpha)=\frac{\Gamma(\tilde{\alpha})}{\Gamma(\alpha)}, \quad \tilde{\alpha}=\frac{d}{2}-\alpha .
$$


It is convenient to rewrite the equation graphically as

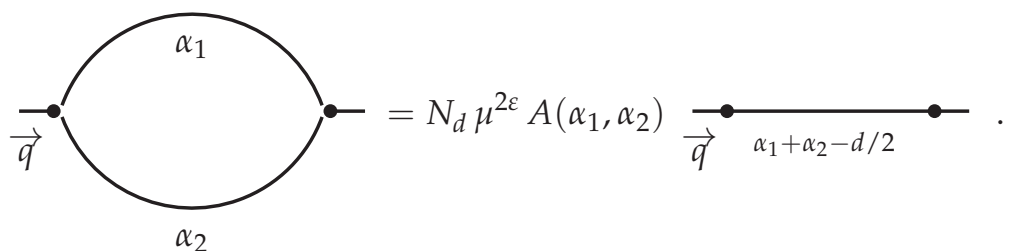

Therefore, all massless diagrams, which can be expressed as combinations of loops and chains, can be evaluated immediately, even when some indices have arbitrary values (see [23-33]). However, starting already with the two-loop level, there are diagrams, which cannot be expressed as combinations of loops and chains (see, for example, Figure 1 in [17]). For these cases there are additional rules.

C. When $\sum \alpha_{i}=d$, there is so-called uniqueness ratio [5-7] for the triangle with indices $\alpha_{i}(i=1,2,3)$

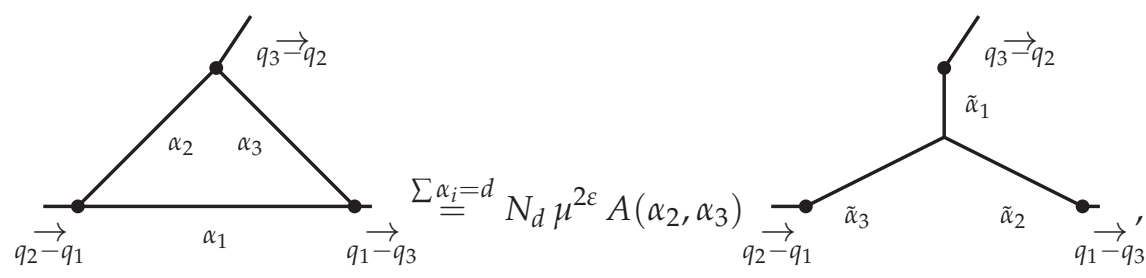

The results (6) can be exactly obtained in the following way: perform an inversion $q_{i} \rightarrow 1 / q_{i}(i=1,2,3), k \rightarrow 1 / k$ in the subintegral expression and in the integral measure. The inversion keeps angles between momenta. After the inversion, one propagator is cancelled because $\sum \alpha_{i}=d$ and the l.h.s. becomes to be equal to a loop. Evaluating it using the rule (5) and returning after it to the initial momenta, we recover the rule (6).

D. For any triangle with indices $\alpha_{i}(i=1,2,3)$ there is the following relation, which is based on integration by parts (IBP) procedure $[6,34,35]$. This paper does not use the IBP procedure for massless charts and, therefore, is not provided here. However, it can be obtained directly from the IBP in the massive case (see (48) below) by setting all masses to zero.

E. Using equation (48) with zero masses allows you to change the indices of the line diagrams by an integer. One can also change line indices using the point group of transformations $[6,36,37]$. The elements of the group are: (a) the transition to momentum presentation, (b) conformal inversion transformation $p \rightarrow p^{\prime}=p / p^{2}$, (c) a special series of transformations that makes it possible to make one of the vertices unique, and then apply the relation (6) to it.

Note that the presence of momenta, and especially the product of momenta in the numerators of the propagators, significantly complicates the FI results and requires generalization of the rules for their calculation (see, for example, [38-41]). However, consideration of this case is beyond the scope of this work.

\section{3. $k R^{\prime}$-Operation}

Calculation of massless diagrams is most important for calculating critical exponents of models and theories, such as anomalous dimensions and $\beta$-functions. One of the most important recipes is the Bogolyubov-Parasyuk-Hepp-Zimmermann (BPHZ) $R$ operation [42-44], which extracts all singularities of any Feynman diagram. Formally, it has the form

$$
R[F I]=F I-k R^{\prime}[F I],
$$

where the $k R^{\prime}$-operation takes into account all the singularities of the subgraphs of the considered diagram, except for the singularities of the diagram itself. A very important 
property of the $k R^{\prime}$-operations is the independence of the result of its application to any $F I$ diagram (i.e., $\left.k R^{\prime}[F I]\right)$ from the external momenta and masses of this FI diagram. This independence is the basis of of infra-red rearrangement approach [45] (see also [46-49]), which allows us to consider $F I$ with the minimum possible set of masses and external momenta: neglecting masses and momenta should not lead to the appearance of infrared singularities. The ability to remove and change external momenta is widely used (see, for example, [50] and references and discussions therein). We show it in our example below, where we will consider in detail the computation of the singular structure of a 5-loop diagram that contributes to the $\beta$-function of the $\varphi^{4}$-model.

To show the applicability of the $k R^{\prime}$-operation, it is convenient to start with the oneloop and two-loop examples.

One loop. Putting $\alpha_{1}=\alpha_{2}=1$ in Equation (5), we have

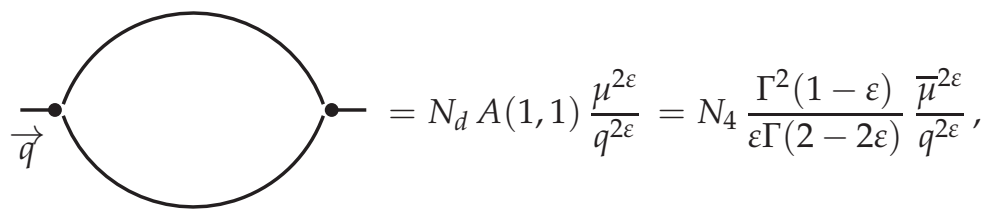

where $\bar{\mu}$ is the scale the $\overline{M S}$-scheme, defined as

$$
\bar{\mu}^{2 \varepsilon}=\mu^{2 \varepsilon}(4 \pi)^{\varepsilon} \Gamma(1+\varepsilon) .
$$

Then $k R^{\prime}$-operation, which in the one-loop case is equal to $k$-operation, because no subgraphs, where $k$-operation is an extraction of the singular part, is the one:

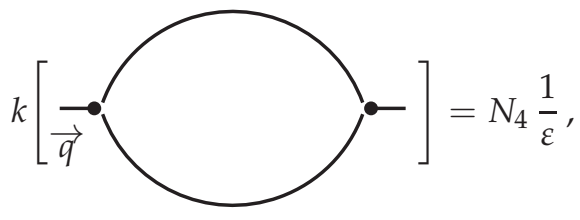

which, of course, is $q^{2}$-independent.

Two loops. Consider the diagram

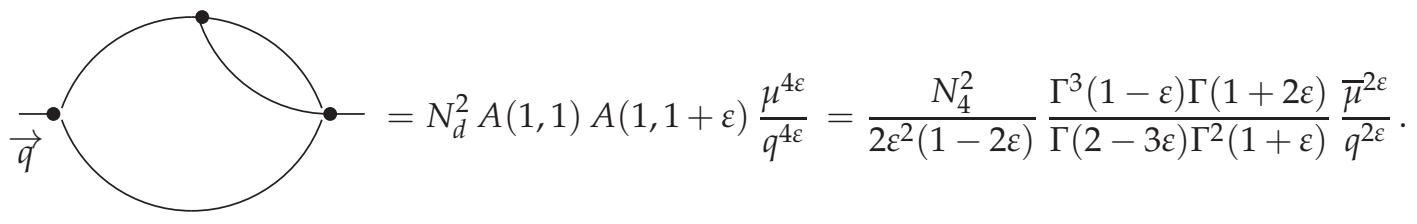

The $R^{\prime}$-operation extracts the singularities of subgraphs. In the considered case, we have the singular internal loop, which singularity is shown in Equation (10). Therefore, the $R^{\prime}$-operation of the considered two-loop diagram has the following form

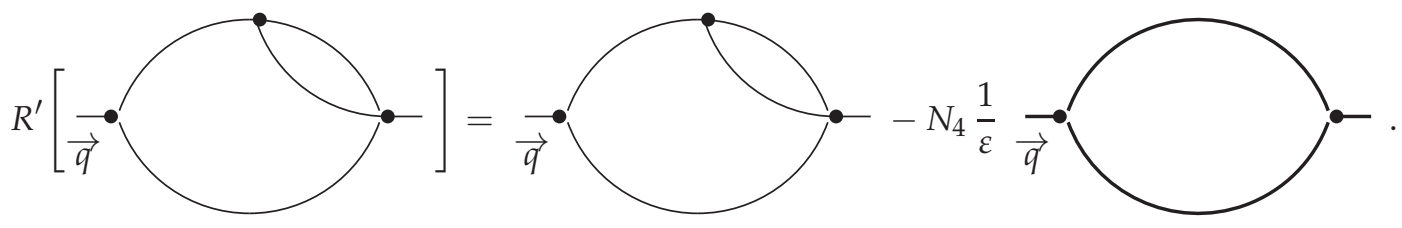

Evaluating loops in the r.h.s. of (12) and taking its singular parts (by the $k$-operation), we have 


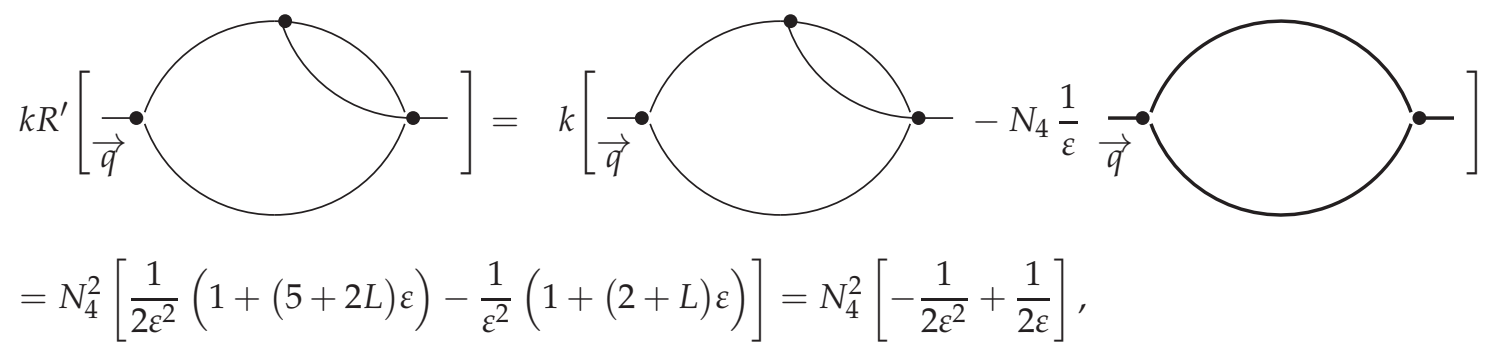

where

$$
L=\ln \left(\frac{\bar{\mu}^{2}}{q^{2}}\right)
$$

As we can see, the r.h.s. of (13) is $q^{2}$-independent.

Fifth loops. Consider the fifth-loop diagram contributed to $\beta$-function of $\varphi^{4}$-model. The $k R^{\prime}$-operation of the diagram has the following form

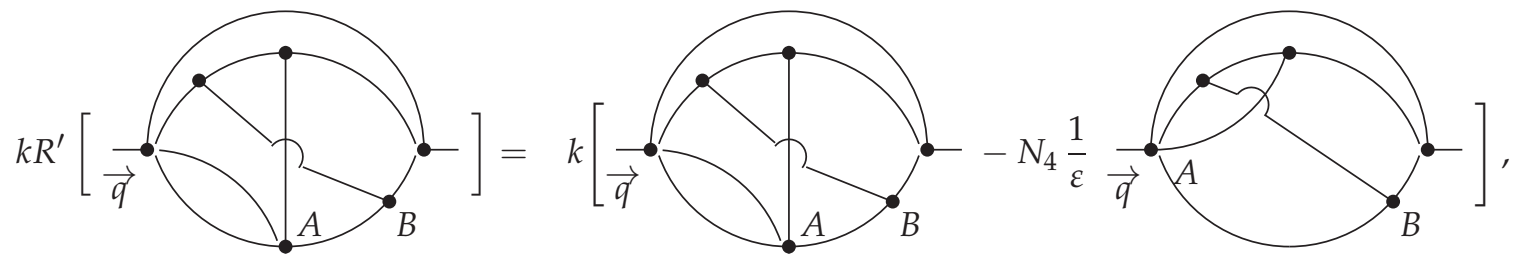

which contains the diagram itself and the counter-term, corresponding the singularity of the internal loop.

Since the right-hand side in (15) is $q^{2}$-independent, we can cancel the external momenta in the considered points and transfer them to the points $\mathrm{A}$ and $\mathrm{B}$ :

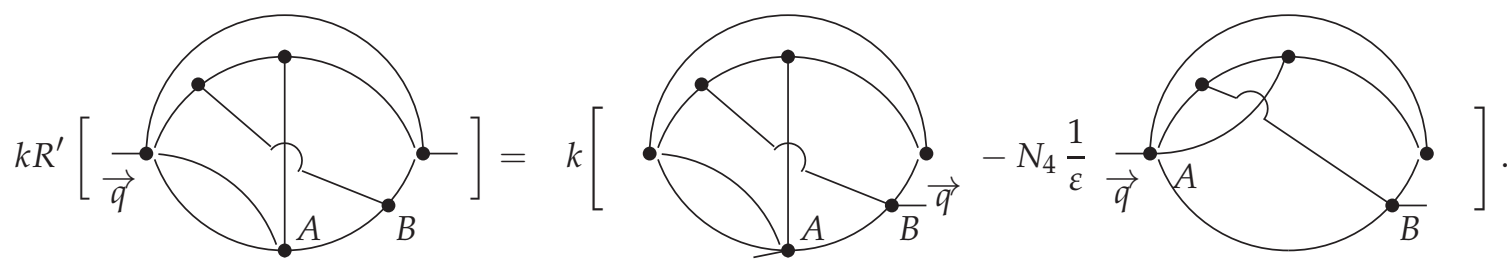

Taking the external momenta in the points A and B as it was done in the r.h.s, we have the following form for the considered diagrams

$$
\underset{q}{A}-\frac{N_{4}}{\varepsilon} \underset{q}{\rightarrow} I_{3}^{B}=N_{d} C_{4} A(1,1+4 \varepsilon)\left(\frac{\mu^{2}}{q^{2}}\right)^{5 \varepsilon}-N_{d} N_{4} \frac{C_{3}}{\varepsilon} A(1,1+3 \varepsilon)\left(\frac{\mu^{2}}{q^{2}}\right)^{4 \varepsilon} \text {, }
$$

where the integrals $I_{4}$ and $I_{3}$ are the internal blocks, which produce the diagrams in the r.h.s. of (16) after integration of the blocks with the propagator between A and B. From the dimensional property, the integrals $I_{4}$ and $I_{3}$ can be represented in the following form

$$
\underset{\vec{q}}{A}=N_{d}^{4} C_{4} \frac{\left(\mu^{2}\right)^{4 \varepsilon}}{\left(q^{2}\right)^{1+4 \varepsilon}}, \underset{I_{3}}{A}=N_{d}^{3} C_{3} \frac{\left(\mu^{2}\right)^{3 \varepsilon}}{\left(q^{2}\right)^{1+3 \varepsilon}}
$$

where $C_{4}$ and $C_{3}$ are the coefficient functions of the integrals $I_{4}$ and $I_{3}$. 
Therefore, we get that

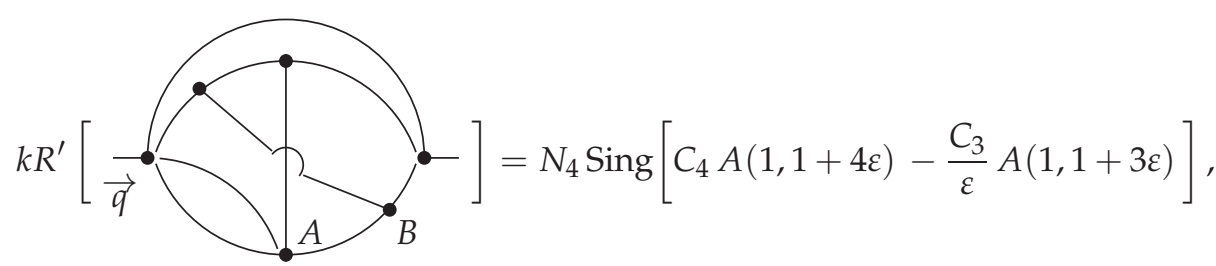

where all $q^{2}$-dependence is cancelled in the r.h.s. singular terms.

For the similar diagram but with the index $1-\varepsilon$ in the line between $A$ and $B$, we have the following

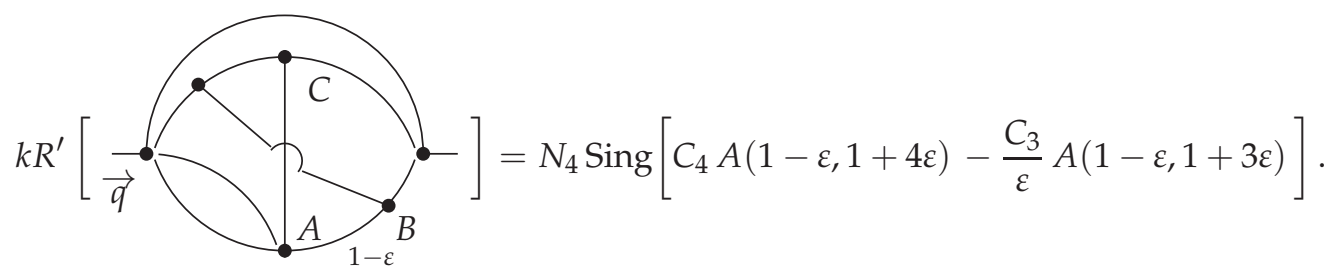

We would like to note that in this diagram we can represent, as an outer line, the line between points $\mathrm{A}$ and $\mathrm{C}$, that is, we get

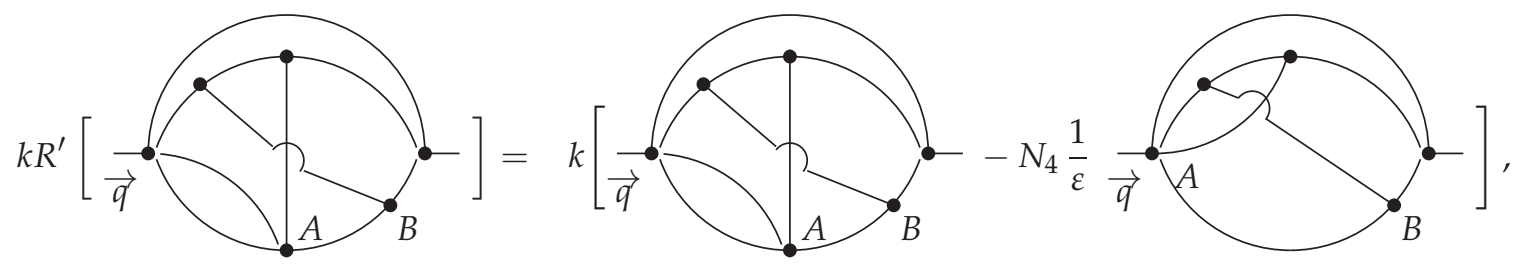

and, thus,

$$
k R^{\prime}
$$

where $C_{4,1}$ and $C_{3,1}$ are the coefficient functions of the integrals $I_{4,1}$ and $I_{3,1}$, which are the internal blocks, which produce the diagrams in the r.h.s. of (21) after integration of the blocks with the propagator between A and C. From the dimensional property, the integrals $I_{4,1}$ and $I_{3,1}$ can be represented in the following form

$$
\underset{\vec{q}}{\stackrel{A}{a} I_{4,1}}=N_{d}^{4} C_{4,1} \frac{\left(\mu^{2}\right)^{4 \varepsilon}}{\left(q^{2}\right)^{1+3 \varepsilon}}, \underset{\vec{q}}{\vec{a}} \stackrel{C}{I_{3,1}}=N_{d}^{3} C_{3,1} \frac{\left(\mu^{2}\right)^{3 \varepsilon}}{\left(q^{2}\right)^{1+2 \varepsilon}},
$$

because they contain one line with the index $1-\varepsilon$.

Now we consider the diagram, similar to the initial one, but with lines between $\mathrm{A}$ and $\mathrm{B}$ and $\mathrm{A}$ and $\mathrm{C}$, having the indices $1-\varepsilon$. Taking as above, the line between $\mathrm{A}$ and $\mathrm{C}$ as an external one, we have the following results 


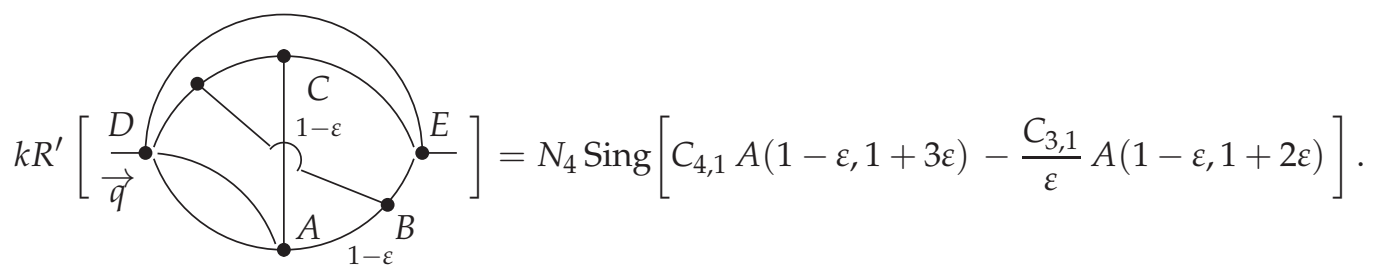

The 1.h.s. diagram, which contains two lines with the index $1-\varepsilon$, can be evaluated exactly. Indeed, we can represent the r.h.s. diagrams as combinations blocks, containing two lines with the index $1-\varepsilon$, and some additional line between $\mathrm{D}$ and $\mathrm{E}$ :

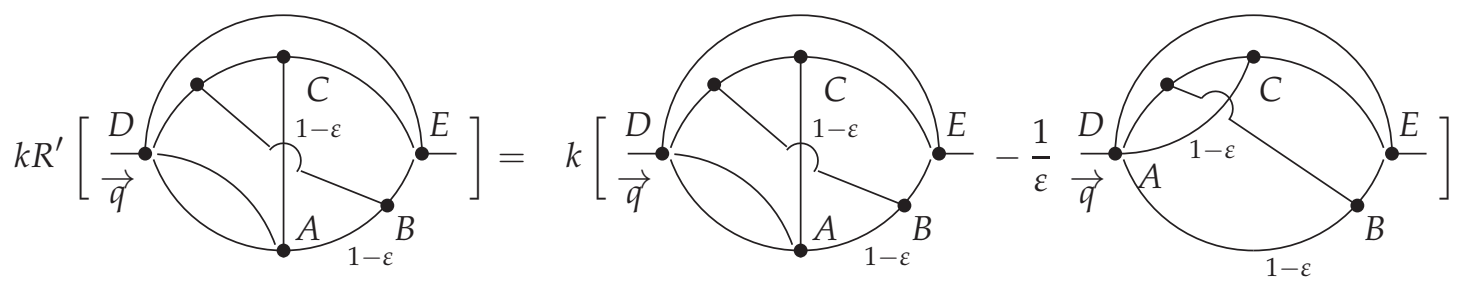

$=N_{d} \operatorname{Sing}\left[C_{4,2} A(1,1+2 \varepsilon)-\frac{C_{3,2}}{\varepsilon} A(1,1+\varepsilon)\right]$,

where $C_{4,2}$ and $C_{3,2}$ are the coefficient functions of the integrals $I_{4,2}$ and $I_{3,2}$, which can be obtained from the diagrams in the r.h.s. taking out the line between D and E. Since they have two lines with the index $1-\varepsilon$, from dimensional properties the integrals $I_{4,2}$ and $I_{3,2}$ can be imagined as

$$
\underset{I_{4,2}}{D}=N_{d}^{4} C_{4,2} \frac{\left(\mu^{2}\right)^{4 \varepsilon}}{\left(q^{2}\right)^{1+2 \varepsilon}}, \frac{D}{\vec{q}}=I_{3,2}^{E}=N_{d}^{3} C_{3,2} \frac{\left(\mu^{2}\right)^{3 \varepsilon}}{\left(q^{2}\right)^{1+\varepsilon}}
$$

Now we consider the integral $I_{4.2}$. After integrating of the internal loop, we have

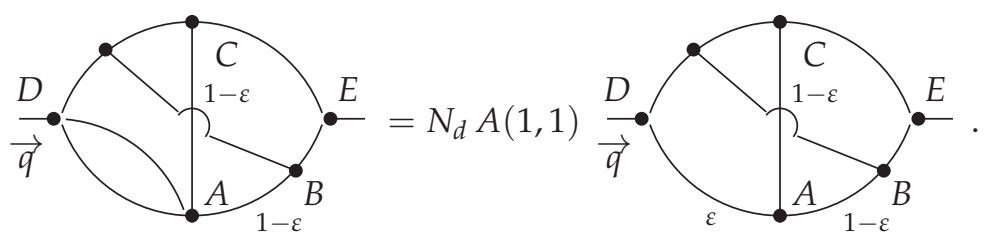

The vertex DAC in the r.h.s. diagram is unical and, thus, it can be replaced by triangle as it was shown in Equation (6). Therefore, we have

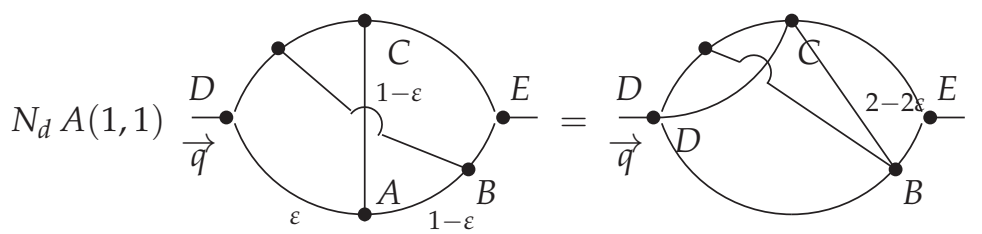


Now the triangle CBE in the r.h.s. diagram is unical and, thus, it can be replaced by vertex in an agreement with (6):
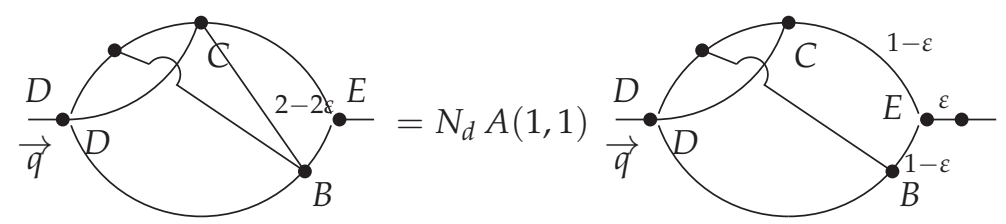

Therefore, finally, we have
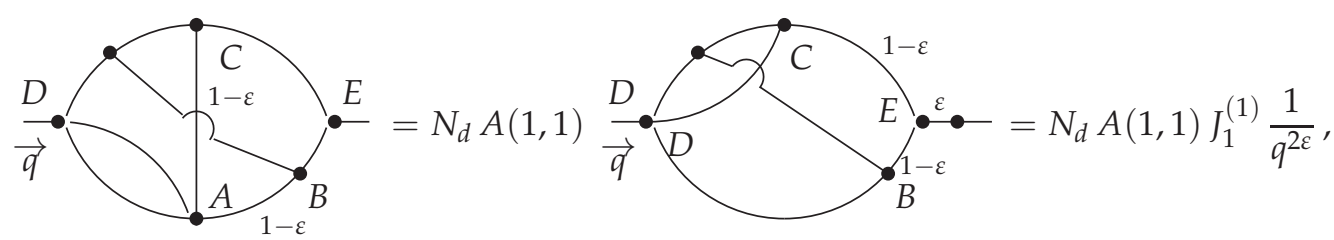

where the integral $J_{1}^{(1)}$ is the table one (see [16]) (Note that the results obtained in [16] are a simple recalculation of the corresponding results $[7,8,12,13]$ found in the $x$-space. For recalculation, it is convenient to use the concept of the so-called dual diagrams (see, for example, [38-41]), which are obtained from the original ones by replacing all momenta with coordinates. With this replacement, the results of the FIs themselves remain unchanged, and only their graphical representation changes. In general, dual diagrams are used [38-41]) in the massless case, but they can also be used [51-53] for propagators with masses.): $J_{1}^{(1)}=J_{1}(1,1,1,1,1,1-\varepsilon, 1-\varepsilon)$. Here

$$
J_{1}\left(a_{1}, a_{2}, a_{3}, a_{4}, a_{5}, a_{6}, a_{7}\right)\left(q^{2}\right)=\vec{q} \overbrace{a_{3}}^{a_{2}}-N_{a_{6}}^{a_{6}} C_{1}^{a_{6}}\left(a_{1}, a_{2}, a_{3}, a_{4}, a_{5}, a_{6}, a_{7}\right) \frac{\left(\bar{\mu}^{2}\right)^{3 \varepsilon}}{\left(q^{2}\right)^{a-3 d / 2}},
$$

where $a=\sum_{k=1}^{7} a_{i}$, and

$$
C_{1}\left(a_{1}, a_{2}, a_{3}, a_{4}, a_{5}, a_{6}, a_{7}\right)=\frac{1}{1-2 \varepsilon}\left[B_{0} \zeta_{5}+\left(B_{1} \zeta_{6}+B_{2} \zeta_{3}^{3}\right) \varepsilon+O\left(\varepsilon^{2}\right)\right]
$$

with

$$
a_{i}=1+\bar{a}_{i} \varepsilon, \quad B_{0}=20, \quad B_{1}=50, \quad B_{2}=20+6 \sum_{k=4}^{7} \bar{a}_{i}
$$

It is convenient to write $C_{1}^{(1)}=C_{1}(1,1,1,1,1,1-\varepsilon, 1-\varepsilon)$ as

$$
C_{1}^{(1)}=C_{1}^{(0)}\left(1-\frac{3 \zeta_{3}^{2}}{5 \zeta_{5}} \varepsilon\right),
$$

where

$$
C_{1}^{(0)}=C_{1}(1,1,1,1,1,1,1)=\frac{10}{1-2 \varepsilon}\left[2 \zeta_{5}+\left(5 \zeta_{6}+2 \zeta_{3}^{3}\right) \varepsilon+O\left(\varepsilon^{2}\right)\right]
$$

The counter-terms $I_{3}\left(q^{2}\right), I_{3,1}\left(q^{2}\right)$ and $I_{3,2}\left(q^{2}\right)$ can be expressed also though $J_{1}\left(a_{1}, a_{2}, a_{3}, a_{4}, a_{5}, a_{6}, a_{7}\right)$ in the following from:

$$
I_{3,2}\left(q^{2}\right)=J_{1}(1,1-\varepsilon, 1-\varepsilon, 1,1,1,1), I_{3,1}\left(q^{2}\right)=J_{1}(1-\varepsilon, 1,1,1,1,1,1), I_{3}\left(q^{2}\right)=J_{1}(1,1,1,1,1,1,1) .
$$


Therefore, within the accuracy $O\left(\varepsilon^{2}\right)$ their coefficient functions are coincide:

$$
C_{3}=C_{3,1}+O\left(\varepsilon^{2}\right)=C_{3,2}+O\left(\varepsilon^{2}\right)=C_{1}^{(0)}+O\left(\varepsilon^{2}\right) .
$$

Taking into account above relations and the results for the one-loop results $A(\alpha, \beta)$, it is possible to show that within the accuracy $O\left(\varepsilon^{2}\right)$ the results for four-loop coefficient functions are also coincide. Indeed,

$$
\begin{aligned}
& C_{4,1}=C_{4,2} \frac{A(1,1+2 \varepsilon)}{A(1-\varepsilon, 1+3 \varepsilon)}-\frac{C_{3,1}}{\varepsilon} \frac{e^{(-L \varepsilon)}}{A(1-\varepsilon, 1+3 \varepsilon)}[A(1,1+\varepsilon)-A(1-\varepsilon, 1+2 \varepsilon)]+O\left(\varepsilon^{2}\right), \\
& C_{4}=C_{4,1} \frac{A(1,1+3 \varepsilon)}{A(1-\varepsilon, 1+4 \varepsilon)}-\frac{C_{3}}{\varepsilon} \frac{e^{(-L \varepsilon)}}{A(1-\varepsilon, 1+4 \varepsilon)}[A(1,1+2 \varepsilon)-A(1-\varepsilon, 1+3 \varepsilon)]+O\left(\varepsilon^{2}\right)
\end{aligned}
$$

and the terms $\sim C_{3,1}$ and $\sim C_{3}$ are suppressed and we have

$$
C_{4}=C_{4,1}+O\left(\varepsilon^{2}\right)=C_{4,2}+O\left(\varepsilon^{2}\right)
$$

and, thus,

$$
C_{4}=C_{1}^{(1)}+O\left(\varepsilon^{2}\right)=C_{1}^{(0)}\left(1+\frac{3 \zeta_{3}^{2}}{10 \zeta_{5}} \varepsilon\right)+O\left(\varepsilon^{2}\right)
$$

Therefore, for the initial diagrams, shown in the r.h.s of Equation (15), using the r.h.s of (19), we have

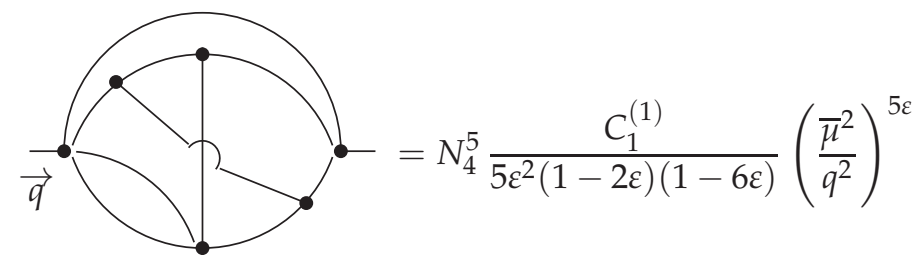

and

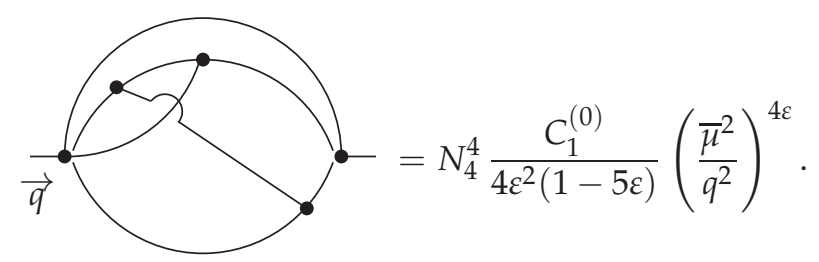

Therefore, for the initial combination shown in (15), we have

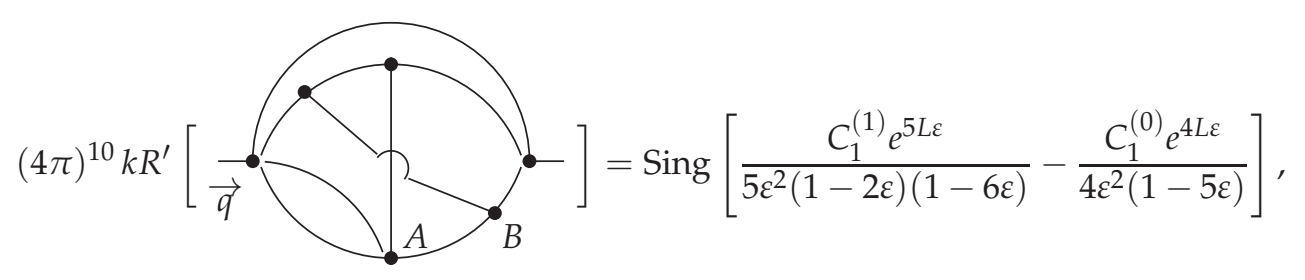

where $L$ is give in Equation (14). Taking the results for $C_{1}^{(1)}$ and $C_{1}^{(0)}$, given in Equations (34) and (35), respectively, we have

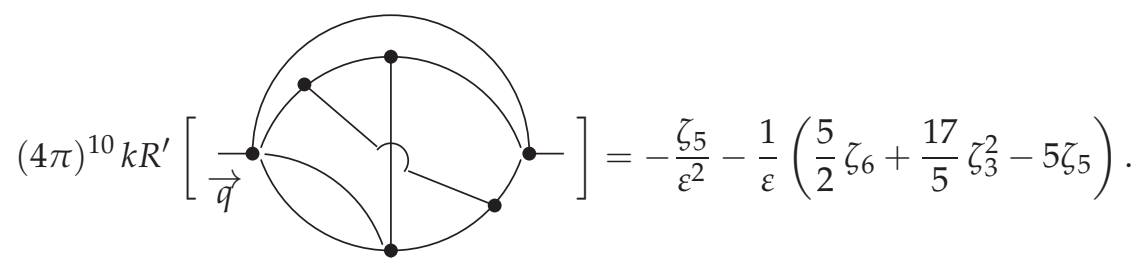




\section{Calculation of Massive Feynman Integrals (Basic Formulas)}

Feynman integrals with massive propagators are significantly more complicated objects compared to the massless case (see, for example, the recent review [54]). The basic rules for calculating such diagrams are discussed in Section 2, which are supplemented by new ones containing directly massive propagators.

Let us briefly consider the rules for calculating diagrams with the massive propagators.

Propagator with mass $M$ is represented as

$$
\frac{1}{\left(q^{2}+M^{2}\right)^{\alpha}}=\underset{\vec{q}}{\stackrel{a}{a}} \quad \text { a . }
$$

The following formulas hold:

A. For simple chain of two massive propagators with the same mass, we have

$$
\frac{1}{\left(q^{2}+M^{2}\right)^{\alpha_{1}}} \frac{1}{\left(q^{2}+M^{2}\right)^{\alpha_{2}}}=\frac{1}{\left(q^{2}+M^{2}\right)^{\left(\alpha_{1}+\alpha_{2}\right)}}
$$

or graphically

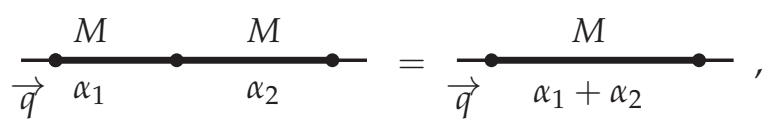

i.e., the product of propagators with the same mass $M$ is equivalent to a new propagator with the mass $M$ and an index equal to the sum of the indices of the original propagators.

B. Massive tadpole is integrated as

$$
\int \frac{D k}{k^{2 \alpha_{1}}\left(k^{2}+M^{2}\right)^{\alpha_{2}}}=N_{d} \frac{R\left(\alpha_{1}, \alpha_{2}\right)}{M^{2\left(\alpha_{1}+\alpha_{2}-d / 2\right)}}
$$

where

$$
R(\alpha, \beta)=\frac{\Gamma\left(d / 2-\alpha_{1}\right) \Gamma\left(\alpha_{1}+\alpha_{2}-d / 2\right)}{\Gamma(d / 2) \Gamma\left(\alpha_{2}\right)} .
$$

C. A simple loop of two massive propagators with masses $M_{1}$ and $M_{2}$ can be represented as hypergeometric function, which can be calculated in a general form, for example, by Feynman-parameter method. With this approach, it is very convenient to represent the loop as the integral of the propagator with the "effective mass" $\mu$ [55-67]:

$$
\begin{aligned}
& (4 \pi)^{d / 2} \times \int \frac{D k}{\left[(q-k)^{2}+M_{1}^{2}\right]^{\alpha_{1}}\left[k^{2}+M_{2}^{2}\right]^{\alpha_{2}}} \\
& =\frac{\Gamma\left(\alpha_{1}+\alpha_{2}-d / 2\right)}{\Gamma\left(\alpha_{1}\right) \Gamma\left(\alpha_{2}\right)} \int_{0}^{1} \frac{d s s^{\alpha_{1}-1}(1-s)^{\alpha_{2}-1}}{\left[s(1-s) q^{2}+M_{1}^{2} s+M_{2}^{2}(1-s)\right]^{\alpha_{1}+\alpha_{2}-d / 2}} \\
& =\frac{\Gamma\left(\alpha_{1}+\alpha_{2}-d / 2\right)}{\Gamma\left(\alpha_{1}\right) \Gamma\left(\alpha_{2}\right)} \int_{0}^{1} \frac{d s}{s^{1-\tilde{\alpha}_{2}}(1-s)^{1-\tilde{\alpha}_{1}}} \frac{1}{\left[q^{2}+\mu^{2}\right]^{\alpha_{1}+\alpha_{2}-d / 2}}, \quad\left(\mu^{2}=\frac{M_{1}^{2}}{1-s}+\frac{M_{2}^{2}}{s}\right) .
\end{aligned}
$$

It is useful to rewrite the equation graphically as

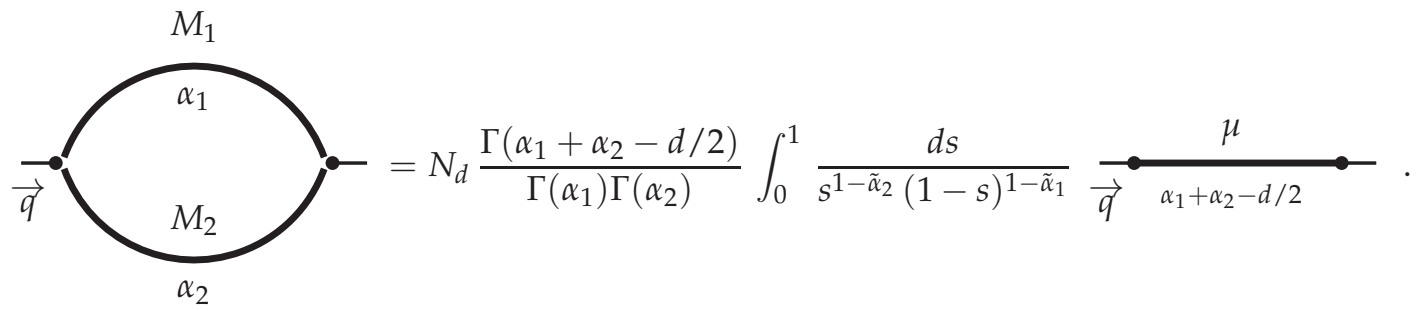


D. For any triangle with indices $\alpha_{i}(i=1,2,3)$ and masses $M_{i}$ there is the following relation, which is based on integration by parts (IBP) procedure $[6,34,35,57-59]$

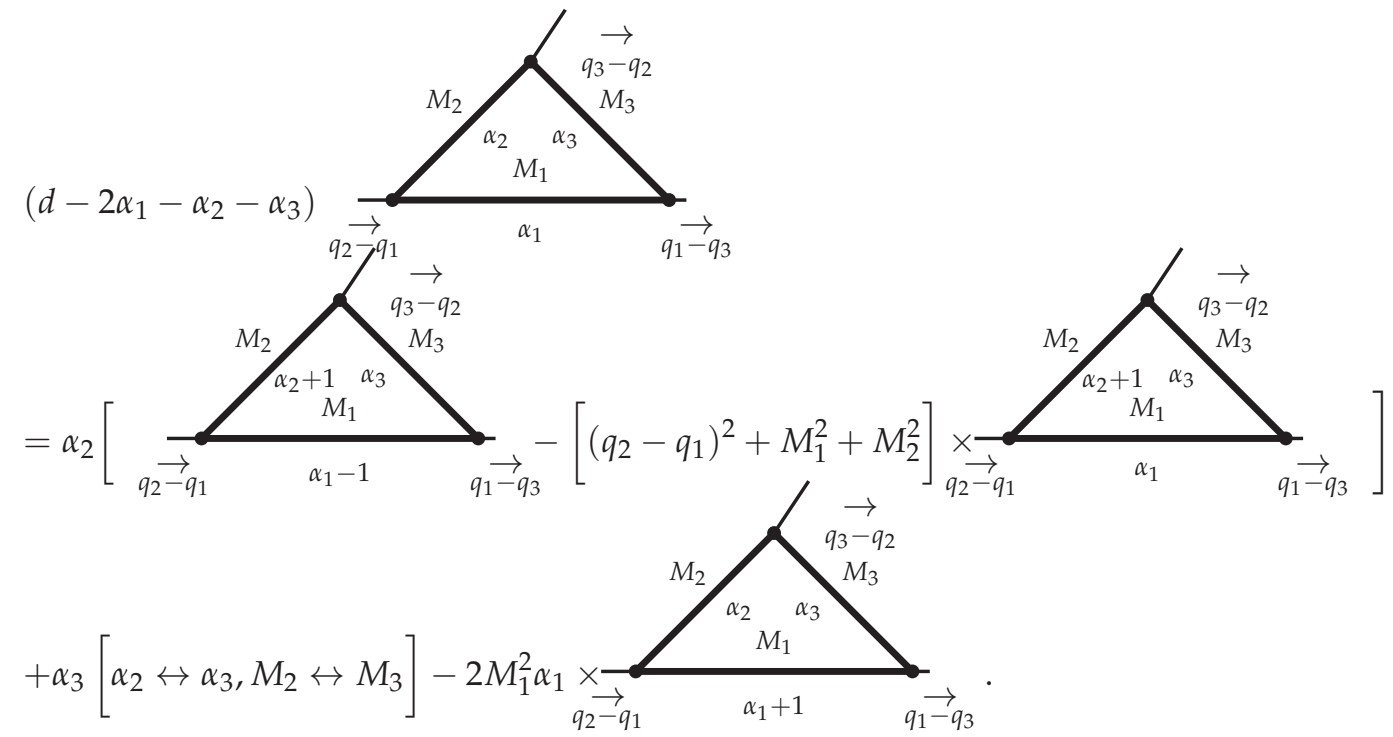

Equation (48) can be obtained by entering the factor $\left(\partial / \partial k_{\mu}\right)\left(k-q_{1}\right)^{\mu}$ into the triangle integrand, shown below as [...]. Indeed, using the procedure of integration by parts, we obtain

$$
\begin{aligned}
& d \int D k[\ldots]=\int D k\left(\frac{\partial}{\partial k_{\mu}}\left(k-q_{1}\right)^{\mu}\right)[\ldots]=\int D k \frac{\partial}{\partial k_{\mu}}\left(\left(k-q_{1}\right)^{\mu}[\ldots]\right) \\
& -\int D k\left(k-q_{1}\right)^{\mu} \frac{\partial}{\partial k_{\mu}}([\ldots])
\end{aligned}
$$

The first term in the r.h.s. becomes to be zero because it can be represented as a surface integral on the infinite surface. Evaluating the second term in the r.h.s. we reproduce Equation (48).

As it is possible to see from Equations (48) and (49) the line with the index $\alpha_{1}$ is distinguished. The contributions of the other lines are the same. Therefore, we will call below the line with the index $\alpha_{1}$ as a "distinguished line". It is clear that a various choices of the distinguished line produce different types of the IBP relations.

The IBP relations lead to differential equations [57-59,68-72] for the considered diagrams (see an example in Section 5) with inhomogeneous terms containing simpler diagrams, i.e., diagrams containing fewer propagators. By repeating the IBP procedure several times, in the last step we can obtain an inhomogeneous term containing only very simple diagrams that can be computed using the $\mathrm{A}-\mathrm{C}$ rules discussed above, as well as the rules discussed in Section 2. By integrating successively the terms in all other inhomogeneous terms, in the last step we can get the result for the original diagram.

E. I would also like to note the importance of the inverse-mass expansions of massive FIs depending on one mass (or on two masses in the on-shall case). The structure of the coefficients of such expansions often has some universality, preserving the complexity (or rang) of harmonic (or nested) sums [73,74] (a more detailed discussion can be found in a recent review [75]). This property simplifies the structure of the results (and the corresponding ansatz for it), and also makes it possible to predict the unknown terms of the expansion. This property is associated with a specific form of differential equations (see discussions in [76-80]) and is most successfully used in the so-called canonical approach [71,72], which is currently the most popular.

Note also that a similar property (in reality, even more strict) takes place in the $N=4$ super Yang-Mills (SYM) model not only for some master integrals, but for the 
kernel of the Balitsky-Fadin-Lipatov-Kuraev (BFKL) equation [81-88], as well as for the anomalous dimensions contributed to the Dokshitzer-Gribov-Lipatov-Altarelli-Parisi (DGLAR) equations [89-93], and for Wilson coefficient functions (see, respectively, [94-99]). This property was called [94] the principle of maximal transcendentality and allows us to obtain anomalous dimensions of Wilson operators and Wilson coefficient functions without any calculations, directly from the corresponding QCD results (if they exist). Moreover, this property (together with the rules $[100,101]$ for analytic continuation) allows predicting the ansatz [102,103] for finding the solution of the corresponding Bethe-ansatz [104-106] and obtaining results for anomalous dimensions in high orders of perturbation theory (see, respectively, [95-98,107-111]. Thus, the anomalous dimensions of the Wilson operators were found [111] in the seven-loop approximation.

\section{Two-Loop on-Shall Master Integral}

Consider the two-loop on-shall master integral (with $q^{2}=-m^{2}$ )

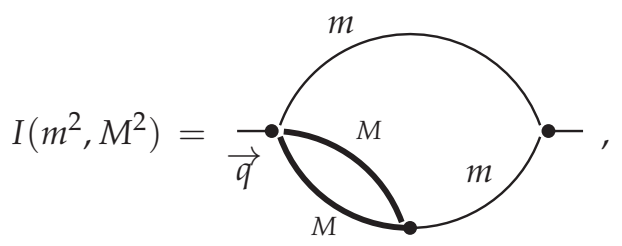

contributing to the $\alpha_{s}$-correction to the ratio between the pole and $\overline{M S}$ masses of the Higgs boson in the standard model.

Except for special places, below we will not indicate the masses $m$ and $M$, but will use rather thin and thick lines for propagators with $m$ and $M$, respectively.

Applying the IBP relation for the inner loop of the integral $I\left(\mathrm{~m}^{2}, \mathrm{M}^{2}\right)$, we have

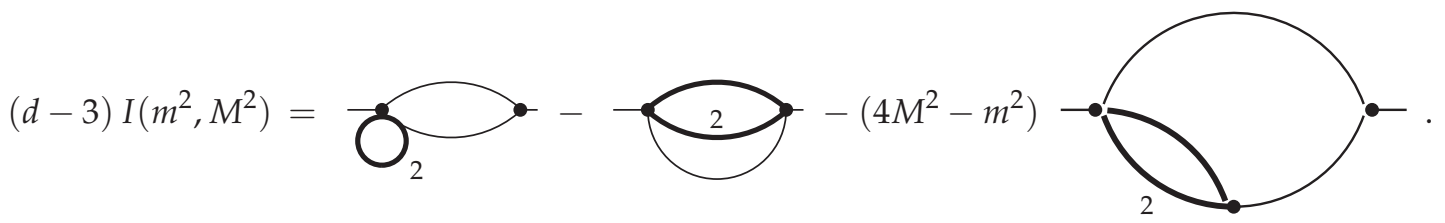

It is possible to see that the last integral in the r.h.s. can be represented as

$$
-\frac{1}{2} \frac{\partial}{\partial M^{2}} I\left(m^{2}, M^{2}\right)
$$

and, thus, Equation (51) can be rewritten as the differential equation

$$
\left(4 M^{2}-m^{2}\right) \frac{1}{2} \frac{\partial}{\partial M^{2}} I\left(m^{2}, M^{2}\right)=(d-3) I\left(m^{2}, M^{2}\right)+J\left(m^{2}, M^{2}\right),
$$

where the inhomogeneous term

$$
J\left(m^{2}, M^{2}\right)=\longrightarrow 2
$$

contains only less complicated diagrams. The solution of the equation with the boundary condition $T\left(M^{2} \rightarrow \infty, m^{2}\right)=0$ has the following form

$$
I\left(M^{2}, m^{2}\right)=N_{4}^{2} \bar{I}(x) \frac{\left(\bar{\mu}^{2}\right)^{2 \varepsilon}}{\left(m^{2}\right)^{2 \varepsilon}}, \quad J\left(M^{2}, m^{2}\right)=N_{4}^{2} \bar{J}(x) \frac{\bar{\mu}^{2}}{\left(m^{2}\right)^{2 \varepsilon}},
$$

$$
\bar{I}(x)=-(4 x-1)^{1 / 2-\varepsilon} \int_{x}^{\infty} \frac{2 \bar{I}_{1}\left(x_{1}\right) d x_{1}}{\left(4 x_{1}-1\right)^{3 / 2-\varepsilon}}=-\frac{(4-z)^{1 / 2-\varepsilon}}{z^{1 / 2-\varepsilon}} \int_{0}^{z} \frac{2 \bar{J}\left(z_{1}\right) d z_{1}}{z_{1}^{1 / 2+\varepsilon}\left(4-z_{1}\right)^{(3 / 2-\varepsilon)}}
$$


where

$$
x=\frac{M^{2}}{m^{2}}, z=\frac{M^{2}}{m^{2}}=\frac{1}{x} .
$$

5.1. $\bar{J}(x)$

The result for the first diagram of $\bar{J}(x)$ can be written in the following form [112,113]:

$$
2=N_{4}^{2} \frac{\left(\bar{\mu}^{2}\right)^{2 \varepsilon}}{\left(M^{2}\right)^{2 \varepsilon}}\left[\frac{1}{2 \varepsilon^{2}}+\frac{1}{2 \varepsilon}-\frac{1}{2}+\ln z+\frac{1-z}{z} \overline{\operatorname{Li}}_{2}(z)\right] \text {, }
$$

where

$$
\overline{\operatorname{Li}}_{2}(z)=\operatorname{Li}_{2}(z)+\ln z \ln (1-z)
$$

and $\operatorname{Li}_{2}(z)$ is dilogarithm [114] (more complicated functions can be found in Refs. [115-117]).

The result for off-shall one-loop can be presented in the form (by using, for example, the rule C)

$\underset{\vec{q}}{\overrightarrow{2}}=\frac{N_{4}}{(1-2 \varepsilon)} \frac{\left(\bar{\mu}^{2}\right)^{\varepsilon}}{\left(m^{2}\right)^{\varepsilon}}\left[\frac{1}{\varepsilon}+\frac{1+y}{1-y}\left[\ln y+\varepsilon\left(\frac{1}{2} \ln ^{2} y-2 \ln y \ln (1+y)-2 \operatorname{Li}_{2}(-y)-\zeta_{2}\right)\right]\right]$,

where $y$ is so-called conformal variable (see Appendix A with the substitution $m^{2} \rightarrow-q^{2}$ ), which is very convenient in the case of massive FIs (see, for example, $[55,56,66,118]$ )

Taking on-shall limit (see also Appendix A), i.e.,

$$
z=1, y=\frac{\sqrt{3}+i}{\sqrt{3}+i}, \frac{1+y}{1-y}=\frac{\sqrt{3}}{i}, \ln y=-\frac{\pi i}{3}, \operatorname{Li}_{2}(-y)=-\frac{1}{3} \zeta_{2}+\frac{2 i}{3} \mathrm{Cl}_{2}\left(\frac{\pi}{3}\right) i^{2}=-1,
$$

where $\mathrm{Cl}_{2}(\pi / 3)$ is the Clausen function, we have

$$
=\frac{N_{4}}{(1-2 \varepsilon)} \frac{\left(\bar{\mu}^{2}\right)^{\varepsilon}}{\left(m^{2}\right)^{\varepsilon}}\left[\frac{1}{\varepsilon}-a_{1}-a_{2} \varepsilon\right]
$$

with

$$
a_{1}=-\frac{\pi}{\sqrt{3}}, a_{2}=\frac{4}{\sqrt{3}} \mathrm{Cl}_{2}\left(\frac{\pi}{3}\right)-\frac{\pi}{\sqrt{3}} \ln 3 .
$$

Then, the result for the second diagram of $\bar{J}(x)$ can be written in the following form

$$
\widehat{O}=\frac{N_{4}^{2}}{(1-2 \varepsilon)} \frac{\left(\bar{\mu}^{2}\right)^{2 \varepsilon}}{\left(M^{2}\right)^{\varepsilon}\left(m^{2}\right)^{\varepsilon}}\left[\frac{1}{\varepsilon^{2}}-\frac{a_{1}}{\varepsilon}-a_{2}\right] \text {. }
$$

Thus, for $\bar{J}(x)$ we have the following result

$$
\bar{J}(z)=\left[-\frac{1}{2 \varepsilon^{2}}+\left(a_{1}-\frac{3}{2}\right) \frac{1}{\varepsilon}+a_{2}+2 a_{1}-\frac{9}{2}+a_{1} \ln z+\frac{1-z}{z} \overline{\operatorname{Li}}_{2}(z)+\frac{1}{2} \ln ^{2} z\right] .
$$

\section{2. $\bar{I}(x)$}

To find the result for the initial diagram $\bar{I}(x)$, see Equation (56), we have to calculate several integrals. 
The first integrals, which corresponds to $z$-independent part of $\bar{J}(x)$, is very simple:

$$
\bar{I}_{1}(x)=(4 x-1)^{1 / 2-\varepsilon} \int_{x}^{\infty} \frac{d x_{1}}{\left(4 x_{1}-1\right)^{3 / 2-\varepsilon}}=\frac{1}{2(1-2 \varepsilon)} .
$$

Other integrals can be calculated at the accuracy $\varepsilon=0$. The integral $\sim \ln z$ in $\bar{J}(x)$ can be evaluated using integration by parts (A similar application of the integration by parts procedure for integral representations can be found in the recent article [119], where FIs containing elliptic structures were considered.) as

$$
\bar{I}_{2}(x)=(4 x-1)^{1 / 2} \int_{x}^{\infty} \frac{d x_{1}}{\left(4 x_{1}-1\right)^{3 / 2}} \ln \left(\frac{1}{x_{1}}\right)=\frac{1}{2}\left[\ln \left(\frac{1}{x}\right)-\tilde{I}(x)\right],
$$

where (see Appendix A)

$$
\begin{aligned}
& \tilde{I}(x)=(4 x-1)^{1 / 2} \int_{x}^{\infty} \frac{d x_{1}}{x_{1}\left(4 x_{1}-1\right)^{1 / 2}}=\frac{(4-z)^{1 / 2}}{z^{1 / 2}} \int_{0}^{z} \frac{d z_{1}}{z_{1}^{1 / 2}\left(4-x_{1}\right)^{1 / 2}} \\
& =\frac{2}{t} \int_{0}^{t} \frac{d t_{1}}{1+t_{1}^{2}}=-\frac{1+y}{1-y} \ln y
\end{aligned}
$$

and, thus,

$$
\bar{I}_{2}(x)=\frac{1}{2}\left[\ln z+\frac{1+y}{1-y} \ln y\right] .
$$

The integral $\sim \ln ^{2} z$ in $\bar{J}(x)$ can be evaluated using integration by parts similarly to the previous one. We have

$$
\bar{I}_{3}(x)=(4 x-1)^{1 / 2} \int_{x}^{\infty} \frac{d x_{1}}{\left(4 x_{1}-1\right)^{3 / 2}} \ln ^{2}\left(\frac{1}{x_{1}}\right)=\frac{1}{2}\left[\ln ^{2}\left(\frac{1}{x}\right)+2 \tilde{I}_{1}(x)\right],
$$

where (see Appendix A)

$$
\begin{aligned}
& \tilde{I}_{1}(x)=(4 x-1)^{1 / 2} \int_{x}^{\infty} \frac{d x_{1}}{x_{1}\left(4 x_{1}-1\right)^{1 / 2}} \ln x_{1}=-\frac{(4-z)^{1 / 2}}{z^{1 / 2}} \int_{0}^{z} \frac{d z_{1}}{z_{1}^{1 / 2}\left(4-x_{1}\right)^{1 / 2}} \ln z_{1} \\
& =-\frac{2}{t} \int_{0}^{t} \frac{d t_{1}}{1+t_{1}^{2}} \ln \left[z_{1}\left(t_{1}\right)\right]=-\frac{1+y}{1-y} \int_{y}^{1} \frac{d y_{1}}{y_{1}} \ln \left[z_{1}\left(y_{1}\right)\right] .
\end{aligned}
$$

The last integral can be calculated using integration by parts as

$-\int_{y}^{1} \frac{d y_{1}}{y_{1}} \ln \left[z_{1}\left(y_{1}\right)\right]=\ln y \ln z-\int_{y}^{1} \frac{d y_{1}\left(1+y_{1}\right)}{y_{1}\left(1-y_{1}\right)} \ln y_{1}=\ln y \ln z+\frac{1}{2} \ln ^{2} y+2 \operatorname{Li}_{2}(1-y) \equiv T_{1}(y)$,

and, thus,

$$
\tilde{I}_{1}(x)=\frac{1+y}{1-y} T_{1}(y) \text { and } \bar{I}_{3}(x)=\frac{1}{2} \ln ^{2} z+\frac{1+y}{1-y} T_{1}(y) .
$$

The last term $\sim \overline{\operatorname{Li}}_{2}(z)$ in $\bar{J}(x)$ can be evaluated using integration by parts similarly to the previous ones. It can be represented as

$$
\bar{I}_{4}(x)=(4 x-1)^{1 / 2} \int_{x}^{\infty} \frac{d x_{1}\left(x_{1}-1\right)}{\left(4 x_{1}-1\right)^{3 / 2}} \overline{\operatorname{Li}}_{2}\left(1 / x_{1}\right)=\frac{1}{2}\left[-\frac{2+z}{2 z} \overline{\operatorname{Li}}_{2}(z)+\tilde{I}_{2}(x)\right],
$$


where

$$
\begin{aligned}
& \tilde{I}_{2}(x)=-(4 x-1)^{1 / 2} \int_{x}^{\infty} \frac{d x_{1}\left(x_{1}+1 / 2\right)}{x_{1}\left(4 x_{1}-1\right)^{1 / 2}} \frac{\partial}{\partial x_{1}} \overline{\mathrm{Li}}_{2}\left(1 / x_{1}\right) \\
& =\frac{(4-z)^{1 / 2}}{z^{1 / 2}} \int_{0}^{z} \frac{d z_{1}\left(2+z_{1}\right)}{2 z_{1}^{1 / 2}\left(4-z_{1}\right)^{1 / 2}} \frac{\partial}{\partial z_{1}} \overline{\mathrm{Li}}_{2}\left(z_{1}\right)
\end{aligned}
$$

Since

$$
\frac{\partial}{\partial z} \overline{\operatorname{Li}}_{2}(z)=-\frac{\ln z}{1-z}
$$

we have

$$
\begin{aligned}
& \tilde{I}_{2}(x)=-\frac{(4-z)^{1 / 2}}{z^{1 / 2}} \int_{0}^{z} \frac{d z_{1}\left(2+z_{1}\right)}{2 z_{1}^{1 / 2}\left(4-z_{1}\right)^{1 / 2}} \frac{\ln z_{1}}{1-z_{1}}=-\frac{2}{t} \int_{0}^{t} \frac{d t_{1}\left(1+3 t_{1}^{2}\right)}{\left(1+t_{1}^{2}\right)\left(1-3 t_{1}^{2}\right)} \ln \left[z_{1}\left(t_{1}\right)\right] \\
& =\frac{1}{t} \int_{0}^{t} d t_{1}\left[\frac{1}{1+t_{1}^{2}}-\frac{3}{1-3 t_{1}^{2}}\right] \ln \left[z_{1}\left(t_{1}\right)\right]=-\frac{1}{2} \frac{1+y}{1-y} T_{1}(y)-3 \tilde{I}_{21}(x) .
\end{aligned}
$$

Now, we study the term $\tilde{I}_{21}(x)$. Considering the integral

$$
\int_{0}^{t} d t_{1} \frac{1}{1-3 t_{1}^{2}}=-\frac{1}{2 \sqrt{3}} \ln \left(\frac{1-\sqrt{3} t}{1+\sqrt{3} t}\right)
$$

we see an appearance of the new variable

$$
\xi=\frac{1-\sqrt{3} t}{1+\sqrt{3} t}
$$

Using the new variable $\xi$ (see Appendix A), we have for $\tilde{I}_{21}(x)$ :

$$
\tilde{I}_{21}(x)=\frac{1}{2 \sqrt{3} t} \int_{\xi}^{1} \frac{d \xi_{1}}{\xi_{1}} \ln \left(\frac{\left(1-\xi_{1}\right)^{2}}{\left(1+\xi_{1}+\xi_{1}^{2}\right)}\right)
$$

Since

$$
\frac{\left(1-\xi_{1}\right)^{2}}{\left(1+\xi_{1}+\xi_{1}^{2}\right)}=\frac{\left(1-\xi_{1}\right)^{3}}{\left(1-\xi_{1}^{3}\right)} \text { and } \frac{1}{\sqrt{3} t}=\frac{1+\xi}{1-\xi^{\prime}}
$$

we can evaluate the integral $\tilde{I}_{21}(x)$ in the following form (We see the appearance of a polynomial structure $\left(1+\xi_{1}+\xi_{1}^{2}\right)$ in the integrand (80), which leads to the appearance of the poly-logarithms with the argument $\xi^{3}$ below in (82). A similar polynomial structure has already been developed, for example, in $[55,56,67,120-124]$ and in the more complicated cases the structure leads to the appearance of the cyclotronic poly-logarithms [125-127].)

$$
\tilde{I}_{21}(x)=\frac{1+\xi}{2(1-\xi)}\left[3 \operatorname{Li}_{2}(\xi)-\frac{1}{3} \operatorname{Li}_{2}\left(\xi^{3}\right)-\frac{8}{3} \zeta_{2}\right] \equiv \frac{1+\xi}{2(1-\xi)} T_{2}(\xi)
$$

and, thus,

$$
\begin{aligned}
& \tilde{I}_{2}(x)=-\frac{1+y}{2(1-y)} T_{1}(y)-\frac{3(1+\xi)}{2(1-\xi)} T_{2}(\xi) \\
& \bar{I}_{4}(x)=-\frac{2+z}{4 z} \overline{\operatorname{Li}}_{2}(z)-\frac{1+y}{4(1-y)} T_{1}(y)-\frac{3(1+\xi)}{4(1-\xi)} T_{2}(\xi) .
\end{aligned}
$$


Thus, the initial master integral $\bar{I}(x)$ can be expressed as

$$
\begin{aligned}
& \bar{I}(x)=\frac{1}{2}\left(\frac{1}{\varepsilon^{2}}+\frac{5-2 a_{1}}{\varepsilon}+19-8 a_{1}-2 a_{2}\right)-a_{1}\left[\ln z+\frac{1+y}{1-y} \ln y\right]-\frac{1}{2} \ln ^{2} z \\
& +\frac{2+z}{2 z} \overline{\operatorname{Li}}_{2}(z)-\frac{1+y}{2(1-y)} T_{1}(y)+\frac{3(1+\xi)}{2(1-\xi)} T_{2}(\xi) .
\end{aligned}
$$

\section{Conclusions}

In this short review, we have presented the results of calculating some massless and massive Feynman integrals.

In the massless case, we considered a 5-loop master diagram that contributes to the $\beta$-function of the $\varphi^{4}$-model. The results for this diagram were obtained $[7,8,12,13]$ by Dmitry Kazakov, but it published without any intermediate calculations. Our calculations are performed in detail (the other two diagrams were discussed in [16]) and the final result coincides with that obtained by Kazakov.

In the massive case, we considered the computation of one of the master integrals contributing to the relationship between the $\overline{M S}$-mass and the pole-mass of the Higgs boson in the standard model in the limit of heavy Higgs. The results for this master integral contain dilogarithms with unusual arguments.

Funding: This research received no external funding.

Institutional Review Board Statement: Not applicable.

Informed Consent Statement: Not applicable.

Data Availability Statement: Not applicable.

Acknowledgments: The author is grateful to Mikhail Kalmykov for collaboration in the calculation of on-shall diagrams, as well as for the correspondence. He is grateful also to Andrei Pikelner for him help with Axodraw2.

Conflicts of Interest: The author declares no conflict of interest.

\section{Appendix A}

Here we present the sets of the new variables that are useful for integrations in the case of massive diagrams:

$$
\begin{aligned}
& t^{2}=\frac{z}{4-z}, \quad z=\frac{4 t^{2}}{1+t^{2}}, \quad 4-z=\frac{4}{1+t^{2}}, \quad(d z)=\frac{8 t(d t)}{\left(1+t^{2}\right)^{2}} \\
& y=\frac{1-i t}{1+i t^{\prime}}, \quad t=\frac{1-y}{i(1+y)}, \quad 1+t^{2}=\frac{4 y}{(1+y)^{2}}, \quad(d t)=-\frac{2}{i} \frac{(d y)}{(1+y)^{2}}, \frac{(d t)}{1+t^{2}}=-\frac{1}{2 i} \frac{(d y)}{y} \\
& \xi=\frac{1-\sqrt{3} t}{1+\sqrt{3} t}, \quad t=\frac{1}{\sqrt{3}} \frac{1-\xi}{1+\xi}, \quad z=\frac{4 t^{2}}{1+t^{2}}=\frac{(1-\xi)^{2}}{1+\xi+\xi^{2}}=\frac{(1-\xi)^{3}}{1-\xi^{3}} \\
& (d t)=-\frac{2(d y)}{\sqrt{3}(1+y)^{2}}, \frac{(d t)}{1-3 t^{2}}=-\frac{1}{2 \sqrt{3}} \frac{(d \xi)}{\xi} .
\end{aligned}
$$

\section{References}

1. 't Hooft, G.; Veltman, M.J.G. Regularization and Renormalization of Gauge Fields. Nucl. Phys. B 1972, 44, 189-213. [CrossRef]

2. Bollini, C.G.; Giambiagi, J.J. Dimensional Renormalization: The Number of Dimensions as a Regularizing Parameter. Nuovo Cim. B 1972, 12, 20.

3. Cicuta, G.M.; Montaldi, E. Analytic renormalization via continuous space dimension. Lett. Nuovo Cim. 1972, 4, 329-332. [CrossRef]

4. 't Hooft, G. Dimensional regularization and the renormalization group. Nucl. Phys. B 1973, 61, 455-468. [CrossRef]

5. D'Eramo, M.; Parisi G.; Peliti, L. Theoretical predictions for critical exponents at the lambda point of bose liquids. Lett. Nuovo Cim. 1971, 2, 878-880. [CrossRef] 
6. Vasiliev, A.N.; Pismak, Y.M.; Khonkonen, Y.R. 1/N Expansion: Calculation of the exponents eta and Nu in the order $1 / \mathrm{N}^{* * 2}$. For arbitrary number of dimensions. Theor. Math. Phys. 1981, 47, 291-306.

7. Kazakov, D.I. The method of uniqueness, a new powerful technique for multiloop calculations. Phys. Lett. B 1983, 133, 406-410. [CrossRef]

8. Kazakov, D.I. Calculation of feynman integrals by the method of 'uniqueness'. Theor. Math. Phys. 1984, 58, 223. [CrossRef]

9. Usyukina, N.I. Calculation of many loop diagrams of perturbation theory. Theor. Math. Phys. 1983, 54, 78-81. [CrossRef]

10. Belokurov, V.V.; Usyukina, N.I. Calculation of ladder diagrams in arbitrary order. J. Phys. A 1983, 16, 2811. [CrossRef]

11. Belokurov, V.V.; Usyukina, N.I. An algorithm for calculating massless feynman diagrams. Theor. Math. Phys. $1989,79,385-391$.

12. Kazakov, D.I. Multiloop calculations: Method of uniqueness and functional equations. Theor. Math. Phys. 1985, 62, 84. [CrossRef]

13. Kazakov, D.I. Analytical Methods for Multiloop Calculations: Two Lectures on the Method of Uniqueness; JINR preprint JINR-E2-84-410 (JINR Publishing Department); Joint Inst. for Nuclear Research: Moscow, Russia, 1984.

14. Kotikov, A.V. The Gegenbauer polynomial technique: The Evaluation of a class of Feynman diagrams. Phys. Lett. B 1996, 375, 240-248. [CrossRef]

15. Kotikov, A.V.; Teber, S. New Results for a Two-Loop Massless Propagator-Type Feynman Diagram. Theor. Math. Phys. 2018, 194, 284-294. [CrossRef]

16. Kotikov, A.V.; Teber, S. Multi-loop techniques for massless Feynman diagram calculations. Phys. Part. Nucl. 2019, 50, 1-41. [CrossRef]

17. Kotikov, A.V. About calculation of massless and massive Feynman integrals. Particles 2020, 3, 394-443. [CrossRef]

18. Peterman, A. Renormalization Group and the Deep Structure of the Proton. Phys. Rept. 1979, 53, 157-248. [CrossRef]

19. Ryder, L.H. Quantum Field Theory; Cambridge University Press: Cambridge, UK, 1996.

20. Gorishnii, S.G.; Larin, S.A.; Tkachov, F.V.; Chetyrkin, K.G. Five Loop Renormalization Group Calculations in the $g \phi^{4}$ in Fourdimensions Theory. Phys. Lett. B 1983, 132, 351

21. Broadhurst, D.J. The master two loop diagram with masses. Z. Phys. C 1990, 47, 115-124. [CrossRef]

22. Kalmykov, M.Y.; Kotikov, A.V. Joint Institute for Nuclear Research, Dubna, Russia. Unpublished work, 2021.

23. Kotikov, A.V. Critical behavior of 3-D electrodynamics. JETP Lett. 1993, 58, 731.

24. Kotikov, A.V. On the Critical Behavior of (2+1)-Dimensional QED. Phys. Atom. Nucl. 2012, 75, 890. [CrossRef]

25. Broadhurst, D.J.; Kotikov, A.V. Compact analytical form for nonzeta terms in critical exponents at order $1 / \mathrm{N}^{* * 3}$. Phys. Lett. B 1998, 441, 345. [CrossRef]

26. Kotikov, A.V.; Shilin, V.I.; Teber, S. Critical behavior of $(2+1)$-dimensional QED: $1 / \mathrm{N}_{f}$ corrections in the Landau gauge. Phys. Rev. D 2016, 94, 056009; Erratum in 2019, 99, 119901. [CrossRef]

27. Kotikov, A.V.; Teber, S. Critical behavior of $(2+1)$-dimensional QED: $1 / N_{f}$ corrections in an arbitrary nonlocal gauge. Phys. Rev. D 2016, 94, 114011; Addendum in 2019, 99, 059902. [CrossRef]

28. Kotikov, A.V.; Teber, S. Critical Behavior of (2+1)-Dimensional QED: $1 /$ N Expansion. Particles 2020, 3, 345-354. [CrossRef]

29. Teber, S. Electromagnetic current correlations in reduced quantum electrodynamics. Phys. Rev. D 2012, 86, 025005. [CrossRef]

30. Teber, S. Two-loop fermion self-energy and propagator in reduced QED 3,2 . Phys. Rev. D 2014, 89, 067702. [CrossRef]

31. Kotikov, A.V.; Teber, S. Note on an application of the method of uniqueness to reduced quantum electrodynamics. Phys. Rev. D 2013, 87, 087701. [CrossRef]

32. Kotikov, A.V.; Teber, S. Two-loop fermion self-energy in reduced quantum electrodynamics and application to the ultrarelativistic limit of graphene. Phys. Rev. D 2014, 89, 065038. [CrossRef]

33. Teber, S.; Kotikov, A.V. The method of uniqueness and the optical conductivity of graphene: New application of a powerful technique for multiloop calculations. Theor. Math. Phys. 2017, 190, 446-457. [CrossRef]

34. Chetyrkin K.G.; Tkachov, F.V. Integration by parts: The algorithm to calculate beta functions in 4 loops. Nucl. Phys. B 1981, 192, 159-204. [CrossRef]

35. Tkachov, F.V. A theorem on analytical calculability of four loop renormalization group functions. Phys. Lett. B 1981, 100, 65. [CrossRef]

36. Gorishnii S.G.; Isaev, A.P. On an Approach to the Calculation of Multiloop Massless Feynman Integrals. Theor. Math. Phys. 1985, 62, 232. [CrossRef]

37. Broadhurst, D.J. Exploiting the 1.440 Fold Symmetry of the Master Two Loop Diagram. Z. Phys. C 1986, 32, 249-253. [CrossRef]

38. Kazakov, D.I.; Kotikov, A.V. The Method of Uniqueness: Multiloop Calculations in QCD. Theor. Math. Phys. 1988, 73, 1264-1274. [CrossRef]

39. Kazakov, D.I.; Kotikov, A.V. Total $\alpha^{-} s$ Correction to Deep Inelastic Scattering Cross-section Ratio, R $=\sigma^{-} 1 / \sigma^{-} \mathrm{t}$ in QCD. Calculation of Longitudinal Structure Function. Nucl. Phys. B 1988, 307, 721;

Erratum in 1990, 345, 299. [CrossRef]

40. Kazakov, D.I.; Kotikov, A.V. On the value of the alpha-s correction to the Callan-Gross relation. Phys. Lett. B 1992, 291, 171-176. [CrossRef]

41. Kotikov, A.V. The Calculation of Moments of Structure Function of Deep Inelastic Scattering in QCD. Theor. Math. Phys. 1989, 78, 134. [CrossRef]

42. Bogoliubov, N.N.; Parasiuk, O.S. On the Multiplication of the causal function in thequantum theory of fields. Acta Math. 1957, 97, 227. 
43. Hepp, K. Proof of the Bogolyubov-Parasiuk theorem on renormalization. Commun. Math. Phys. 1966, 2, 301-326. [CrossRef]

44. Zimmermann, W. Convergence of Bogoliubov's method of renormalization in momentumspace. Commun. Math. Phys. 1969, 15, 208-243. [CrossRef]

45. Vladimirov, A.A. Method for Computing Renormalization Group Functions in Dimensional Renormalization Scheme. Theor. Math. Phys. 1980, 43, 417. [CrossRef]

46. Chetyrkin, K.G.; Kataev, A.L.; Tkachov, F.V. New Approach to Evaluation of Multiloop Feynman Integrals: The Gegenbauer Polynomial x Space Technique. Nucl. Phys. B 1980, 174, 345-377. [CrossRef]

47. Chetyrkin, K.G.; Tkachov, F.V. Infrared R Operation And Ultraviolet Counterterms in the Ms Scheme. Phys. Lett. 1982, B114, 340-344. [CrossRef]

48. Chetyrkin, K.G.; Smirnov, V.A. R* Operation Corrected. Phys. Lett. 1984, B144, 419-424. [CrossRef]

49. Smirnov, V.A.; Chetyrkin, K.G. R* Operation in the Minimal Subtraction Scheme. Theor. Math. Phys. 1985, 63, 462-469. [CrossRef]

50. Chetyrkin, K.G. Combinatorics of $R, R^{-1}$, and $R^{*}$-operations and asymptotic expansions of feynman integrals in the limit of large momenta and masses. arXiv 1991, arXiv:1701.08627.

51. Kotikov, A.V. New method of massive Feynman diagrams calculation. Mod. Phys. Lett. A 1991, 6, 677-692. [CrossRef]

52. Kotikov, A.V. New method of massive Feynman diagrams calculation. Vertex type functions. Int. J. Mod. Phys. A 1992, 7, 1977. [CrossRef]

53. Henn, J.M.; Plefka, J.C. Scattering Amplitudes in Gauge Theories. Lect. Notes Phys. 2014, 883, 1.

54. Blumlein, J. Analytic integration methods in quantum field theory: An Introduction. arXiv 2021, arXiv:2103.10652 .

55. Fleischer, J.; Kotikov, A.V.; Veretin, O.L. Analytic two loop results for selfenergy type and vertex type diagrams with one nonzero mass. Nucl. Phys. B 1999, 547, 343.

56. Fleischer, J.; Kotikov, A.V.; Veretin, O.L. Applications of the large mass expansion. Acta Phys. Polon. B 1998, $29,2611$.

57. Kotikov, A.V. Differential equations method: New technique for massive Feynman diagrams calculation. Phys. Lett. B 1991, 254, 158-164. [CrossRef]

58. Kotikov, A.V. Differential equations method: The Calculation of vertex type Feynman diagrams. Phys. Lett. B 1991, 259, 314-322. [CrossRef]

59. Kotikov, A.V. Differential equation method: The Calculation of N point Feynman diagrams. Phys. Lett. B 1991, 267, 123-127. [CrossRef]

60. Kotikov, A.V. New method of massive N point Feynman diagrams calculation. Mod. Phys. Lett. A 1991, 6, 3133-3141. [CrossRef]

61. Kniehl, B.A.; Kotikov, A.V.; Onishchenko, A.; Veretin, O. Two-loop sunset diagrams with three massive lines. Nucl. Phys. B 2006, 738, 306-316. [CrossRef]

62. Kniehl, B.A.; Kotikov, A.V.; Onishchenko, A.I.; Veretin, O.L. Two-loop diagrams in non-relativistic QCD with elliptics. Nucl. Phys. B 2019, 948, 114780 [CrossRef]

63. Kniehl B.A.; Kotikov, A.V. Calculating four-loop tadpoles with one non-zero mass. Phys. Lett. B 2006, 638, 531-537. [CrossRef]

64. Kniehl, B.A.; Kotikov, A.V. Counting master integrals: Integration-by-parts procedure with effective mass. Phys. Lett. B 2012, 712, 233-234. [CrossRef]

65. Fleischer, J.; Kotikov, A.V.; Veretin, O.L. The differential equation method: Calculation of vertex-type diagrams with one non-zero mass. Phys. Lett. B 1998, 417, 163-172. [CrossRef]

66. Fleischer, J.; Kalmykov, M.Y.; Kotikov, A.V. Two-loop self-energy master integrals on shell. Phys. Lett. B 1999, 462, 169-177. [CrossRef]

67. Kotikov, A.; Kuhn, J.H.; Veretin, O. Two-Loop Formfactors in Theories with Mass Gap and Z-Boson Production. Nucl. Phys. B 2008, 788, 47-62. [CrossRef]

68. Remiddi, E. Differential equations for Feynman graph amplitudes. Nuovo Cim. A 1997, 110, 1435-1452.

69. Bern, Z.; Dixon, L.J.; Dunbar, D.C.; Kosower, D.A. One loop n point gauge theory amplitudes, unitarity and collinear limits. Nucl. Phys. B 1994, 425, 217-260. [CrossRef]

70. Gehrmann, T.; Remiddi, E. Differential equations for two loop four point functions. Nucl. Phys. B 2000, 580, 485-518. [CrossRef]

71. Henn, J.M. Multiloop integrals in dimensional regularization made simple. Phys. Rev. Lett. 2013, 110, 251601. [CrossRef]

72. Henn, J.M. Lectures on differential equations for Feynman integrals. J. Phys. A 2015, 48, 153001. [CrossRef]

73. Vermaseren, J.A.M. Harmonic sums, Mellin transforms and integrals. Int. J. Mod. Phys. A 1999, 14, 2037-2076. [CrossRef]

74. Blumlein, J.; Kurth, S. Harmonic sums and Mellin transforms up to two loop order. Phys. Rev. D 1999, 60, 014018 [CrossRef]

75. Kotikov, A.V. Differential equations and Feynman integrals. arXiv 2021, arXiv:2102.07424.

76. Kotikov, A.V. The Property of maximal transcendentality in the $\mathrm{N}=4$ Supersymmetric Yang-Mills. arXiv 2010, arXiv:1005.5029.

77. Kotikov, A.V. The property of maximal transcendentality: Calculation of anomalous dimensions in the $\mathcal{N}=4 \mathrm{SYM}$ and master integrals. Phys. Part. Nucl. 2013, 44, 374-385. [CrossRef]

78. Kotikov, A.V.; Onishchenko, A.I. DGLAP and BFKL equations in $\mathcal{N}=4$ SYM: From weak to strong coupling. arXiv 2019, arXiv:1908.05113.

79. Kotikov, A.V. The property of maximal transcendentality: Calculation of master integrals. Theor. Math. Phys. 2013, 176, 913-921. [CrossRef]

80. Kotikov, A.V. The property of maximal transcendentality: Calculation of Feynman integrals. Theor. Math. Phys. 2017, 190, 391-401. [CrossRef] 
81. Lipatov, L.N. Reggeization of the Vector Meson and the Vacuum Singularity in Nonabelian Gauge Theories. Sov. J. Nucl. Phys. 1976, 23, 338.

82. Fadin, V.S.; Kuraev, E.A.; Lipatov, L.N. On the Pomeranchuk Singularity in Asymptotically Free Theories. Phys. Lett. B 1975, 60, 50-52. [CrossRef]

83. Kuraev, E.A.; Lipatov, L.N.; Fadin, V.N. Multi-Reggeon Processes in the Yang-Mills Theory. Sov. Phys. JETP 1976, $44,443$.

84. Kuraev, E.A.; Lipatov, L.N.; Fadin, V.N. The Pomeranchuk Singularity in Nonabelian Gauge Theories. Sov. Phys. JETP 1977, 45, 199.

85. Balitsky, I.I.; Lipatov, L.N. The Pomeranchuk Singularity in Quantum Chromodynamics. Sov. J. Nucl. Phys. 1978, $28,822$.

86. Balitsky, I.I.; Lipatov, L.N. Calculation of meson meson interaction cross-section in quantum chromodynamics. JETP Lett. 1979, 30, 355-386.

87. Fadin, V.S.; Lipatov, L.N. BFKL pomeron in the next-to-leading approximation. Phys. Lett. B 1998, 429, 127-134. [CrossRef]

88. Camici, G.; Ciafaloni, M. Energy scale(s) and next-to-leading BFKL equation. Phys. Lett. B 1998, 430, 349-354.

89. Gribov, V.N.; Lipatov, L.N. Deep inelastic e p scattering in perturbation theory. Sov. J. Nucl. Phys. 1972, 15, 438.

90. Gribov, V.N.; Lipatov, L.N. e+ e- pair annihilation and deep inelastic e p scattering in perturbation theory. Sov. J. Nucl. Phys. 1972, 15, 675 .

91. Lipatov, L.N. The parton model and perturbation theory. Sov. J. Nucl. Phys. 1975, 20, 94.

92. Altarelli, G.; Parisi, G. Asymptotic Freedom in Parton Language. Nucl. Phys. B 1977, 126, 298-318. [CrossRef]

93. Dokshitzer, Y.L. Calculation of the Structure Functions for Deep Inelastic Scattering and e+ e- Annihilation by Perturbation Theory in Quantum Chromodynamics. Sov. Phys. JETP 1977, 46, 641.

94. Kotikov, A.V.; Lipatov, L.N. NLO corrections to the BFKL equation in QCD and in supersymmetric gauge theories. Nucl. Phys. B 2000, 582, 19-43. [CrossRef]

95. Kotikov, A.V.; Lipatov, L.N. DGLAP and BFKL equations in the $\mathrm{N}=4$ supersymmetric gauge theory. Nucl. Phys. B 2003, 661, 19-61. [CrossRef]

96. Kotikov, A.V.; Lipatov, L.N. DGLAP and BFKL evolution equations in the $\mathrm{N}=4$ supersymmetric gauge theory. arXiv 2001, arXiv:hep-ph/0112346.

97. Kotikov, A.V.; Lipatov, L.N.; Velizhanin, V.N. Anomalous dimensions of Wilson operators in N = 4 SYM theory. Phys. Lett. B 2003, 557, 114-120. [CrossRef]

98. Kotikov, A.V.; Lipatov, L.N.; Onishchenko, A.I.; Velizhanin, V.N. Three loop universal anomalous dimension of the Wilson operators in N = 4 SUSY Yang-Mills model. Phys. Lett. B 2004, 595, 521-529. [CrossRef]

99. Bianchi, L.; Forini, V.; Kotikov, A.V. On DIS Wilson coefficients in N = 4 super Yang-Mills theory. Phys. Lett. B 2013, 725, 394-401. [CrossRef]

100. Kotikov, A.V. Gluon distribution for small x. Phys. At. Nucl. 1994, 57, 133.

101. Kotikov, A.V.; Velizhanin, V.N. Analytic continuation of the Mellin moments of deep inelastic structure functions. arXiv 2005, arXiv:hep-ph/0501274.

102. Kotikov, A.V.; Rej, A.; Zieme, S. Analytic three-loop Solutions for N = 4 SYM Twist Operators. Nucl. Phys. B 2009, 813, 460-483. [CrossRef]

103. Beccaria, M.; Belitsky, A.V.; Kotikov, A.V.; Zieme, S. Analytic solution of the multiloop Baxter equation. Nucl. Phys. B 2010, 827, 565-606. [CrossRef]

104. Staudacher, M. The Factorized S-matrix of CFT/AdS. JHEP 2005, 0505, 054. [CrossRef]

105. Beisert, N.; Staudacher, M. Long-range psu(2,2|4) Bethe Ansatze for gauge theory and strings. Nucl. Phys. B 2005, 727, 1-62. [CrossRef]

106. Beisert, N.; Eden, B.; Staudacher, M. Transcendentality and Crossing. J. Stat. Mech. 2007, 0701, P01021. [CrossRef]

107. Kotikov, A.V.; Lipatov, L.N.; Rej, A.; Staudacher, M.; Velizhanin, V.N. Dressing and wrapping. J. Stat. Mech. 2007,0710, P10003. [CrossRef]

108. Bajnok, Z.; Janik, R.A.; Lukowski, T. Four-loop perturbative Konishi from strings and finite size effects for multiparticle states. Nucl. Phys. B 2009, 816, 376. [CrossRef]

109. Lukowski, T.; Rej, A.; Velizhanin, V.N. Five-Loop Anomalous Dimension of Twist-Two Operators. Nucl. Phys. B 2010, 831, 105-132. [CrossRef]

110. Marboe, C.; Velizhanin V.N.; Volin, D. Six-loop anomalous dimension of twist-two operators in planar $\mathcal{N}=4$ SYM theory. JHEP 2015, 1507, 084. [CrossRef]

111. Marboe, C.; Velizhanin, V. Twist-2 at seven loops in planar $\mathcal{N}=4$ SYM theory: Full result and analytic properties. JHEP 2016, 1611, 013. [CrossRef]

112. Gray, N.; Broadhurst, D.J.; Grafe, W.; Schilcher, K. Three Loop Relation of Quark (Modified) Ms And Pole Masses. Z. Phys. C 1990, 48, 673-679. [CrossRef]

113. Argeri, M.; Mastrolia, P.; Remiddi, E. The analytic value of the sunrise self-mass with two equal masses and the external invariant equal to the third squared mass. Nucl. Phys. B 2002, 631, 388-400. [CrossRef]

114. Lewin, L. Polylogarithms and Associated Functions; North Holland: Amsterdam, The Netherlands, 1981.

115. Devoto, A.; Duke, D.W. Table of Integrals and Formulae for Feynman Diagram Calculations. Riv. Nuovo Cim. 1984, 7N6, 1-39. [CrossRef] 
116. Remiddi, E.; Vermaseren, J.A.M. Harmonic polylogarithms. Int. J. Mod. Phys. A 2000, 15, 725-754. [CrossRef]

117. Goncharov, A.B. Periods and mixed motives. arXiv 2002, arXiv:math/0202154.

118. Davydychev, A.I.; Kalmykov, M.Y. Massive Feynman diagrams and inverse binomial sums. Nucl. Phys. B 2004, $699,3-64$. [CrossRef]

119. Campert, J.; Moriello, F.; Kotikov, A. Sunrise integral with two internal masses and pseudo-threshold kinematics in terms of elliptic polylogarithms. arXiv 2020, arXiv:2011.01904.

120. Aglietti, U.; Bonciani, R. Master integrals with one massive propagator for the two loop electroweak form-factor. Nucl. Phys. B 2003, 668, 3-76. [CrossRef]

121. Aglietti, U.; Bonciani, R.; Degrassi, G.; Vicini, A. Two loop light fermion contribution to Higgs production and decays. Phys. Lett. B 2004, 595, 432-441. [CrossRef]

122. Aglietti, U.; Bonciani, R.; Degrassi, G.; Vicini, A. Master integrals for the two-loop light fermion contributions to gg $\longrightarrow>\mathrm{H}$ and $\mathrm{H}$ $\longrightarrow$ gamma gamma. Phys. Lett. B 2004, 600, 57-64. [CrossRef]

123. Aglietti, U.; Bonciani, R.; Degrassi, G.; Vicini, A. Analytic Results for Virtual QCD Corrections to Higgs Production and Decay. JHEP 2007, 1, 21. [CrossRef]

124. Lee, R.N.; Schwartz, M.D.; Zhang, X. Compton Scattering Total Cross Section at Next-to-Leading Order. Phys. Rev. Lett. 2021, 126, 211801. [CrossRef]

125. Blumlein, J.; Schneider, C. Analytic Computing Methods for Precision Calculations in Quantum Field Theory. Int. J. Mod. Phys. A 2018, 33, 1830015. [CrossRef]

126. Ablinger, J.; Blumlein, J.; Schneider, C. Iterated integrals over letters induced by quadratic forms. Phys. Rev. D 2021, $103,096025$. [CrossRef]

127. Ablinger, J.; Blumlein, J.; Schneider, C. Harmonic Sums and Polylogarithms Generated by Cyclotomic Polynomials. J. Math. Phys. 2011, 52, 102301. [CrossRef] 